\title{
Tissue Characterization Using Fractal Dimension of High Frequency Ultrasound RF Time Series
}

\author{
Mehdi Moradi ${ }^{1}$, Parvin Mousavi $^{1}$, and Purang Abolmaesumi ${ }^{1,2}$ \\ ${ }^{1}$ School of Computing, Queen's University, Kingston, Canada \\ 2 Department of Electrical and Computer Engineering, Queen's University \\ \{moradi, pmousavi, purang\}@cs . queensu.ca
}

\begin{abstract}
This paper is the first report on the analysis of ultrasound $\mathrm{RF}$ echo time series acquired using high frequency ultrasound. We show that variations in the intensity of one sample of RF echo over time is correlated with tissue microstructure. To form the RF time series, a high frequency probe and a tissue sample were fixed in position and RF signals backscattered from the tissue were continuously recorded. The fractal dimension of RF time series was used as a feature for tissue classification. Feature values acquired from different areas of one tissue type were statistically similar. For animal tissues with different cellular microstructure, we successfully used the fractal dimension of RF time series to distinguish segments as small as 20 microns with accuracies as high as $98 \%$. The results of this study demonstrate that the analysis of $\mathrm{RF}$ time series is a promising approach for distinguishing tissue types with different cellular microstructure.
\end{abstract}

\section{Introduction}

Ultrasound-based tissue characterization techniques rely on different scattering patterns of ultrasound in tissues with dissimilar cellular microstructures. Although the exact physical mechanisms that govern these patterns are not well understood [1, microstructure-induced differences in ultrasound-tissue interaction are documented both at clinical (2-10 MHz) frequencies 2] and at higher frequencies 13. In other words, ultrasound Radio-Frequency (RF) echoes contain information about tissue characteristics. However, it is challenging to disentangle this information from the variations in the signal caused by the systemdependent effects, such as mechanical and electrical properties of the transducer and diffraction effects due to the finite aperture of the transducer. This fundamental restriction of ultrasound-based tissue characterization techniques limit their sensitivity and specificity in diagnosis of cancer lesions [45].

In a new approach to analysis of RF echoes for tissue characterization, we have recently proposed that if a specific location in tissue undergoes continuous interactions with ultrasound, the time series of the RF echo signals (see Figure1) from that location carries "tissue characterizing" information [6]. In other words, although variations in the intensity of one sample of RF echo over time are partly due to the electronic noise of the ultrasound machine or the errors caused during 

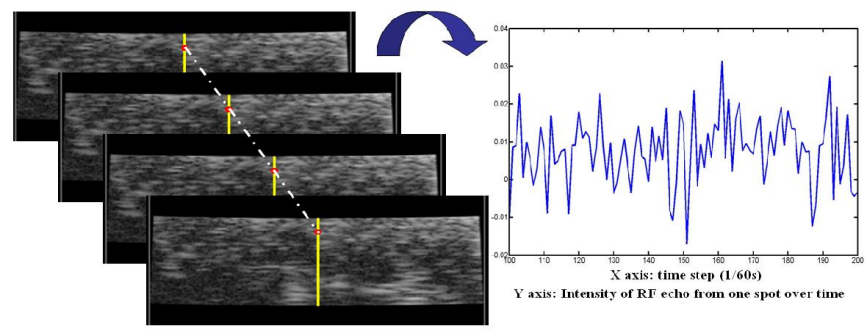

Fig. 1. RF time series: Sequential echoes received from one location of tissue

the beam-forming process [8], they depend on the tissue type as well. Specifically, the fractal dimension (FD) of the RF time series was successfully used to detect cancerous lesions in prostate tissue [7], as well as to distinguish different animal tissues [6].

These tissue characteristic variations might be due to the vibrations in the microstructural level induced by the continuous emission of ultrasound beams. However, more studies on the origin of this phenomenon are necessary. Specifically, in the current paper we report the results of our new research that addresses three fundamental questions about the RF time series: 1) Are the results of tissue classification based on the RF time series, correlated with the microstructure of the tissue? In other words, is this approach more successful in separating tissue types with significantly different microstructures? 2) What is the effect of utilizing high frequency ultrasound on the outcome of the method? It is a well-known fact that at very high frequencies the scattering of ultrasound is primarily caused by the cellular microstructure [1] as opposed to tissue macrostructure. Therefore, the dependence of the FD of RF time series on cellular microstructure should be more evident in high frequency data. 3) Are the tissue characterizing properties of RF time series emphasized in A-mode? Some of the system related alterations in the RF time series acquired in B-mode, including the beam-forming, are absent in A-mode imaging.

To answer these questions, we analyzed RF echo time series acquired using high frequency ultrasound A-mode probes. Based on microscopic studies, we showed that at these frequencies, the separability of tissues based on FD of RF time series is closely related to differences in tissue microstructures. We used FD of the RF times series to successfully distinguish segments as small as 20 microns of animal tissues of dissimilar microstructures with accuracies as high as $98 \%$. Furthermore, the FD values calculated from the RF time series of different tissues showed statistically significant differences, far beyond the variations in FD values in one tissue type. These experiments suggest the presence of microstructurerelated information in the $\mathrm{RF}$ time series. Some of the pathologic conditions, including cancer, are characterized by a dramatic change in the microstructure of the affected tissue. Therefore, the proposed tissue characterization approach could lead to an effective method for diagnosis of certain types of cancer [7]. The rest of this paper is organized as follows: Section 2 introduces the data 
collection method, feature extraction and classification approaches, Section 3 presents our results and discussions, and Section 4 provides a summary and the conclusions.

\section{Methods}

To study the tissue characterizing capabilities of RF echo time series acquired at higher frequencies, we used four different tissue types: bovine liver, pig liver, bovine muscle, and chicken breast. As illustrated in Figure 2, the cellular structure of both bovine and pig liver are characterized by hepatocyte cells (of slightly different shape and density), whereas bovine muscle and chicken breast both have fibrous structures formed by sarcomeres.

The high frequency ultrasound RF time series in this study were collected using a Vevo 770 high resolution ultrasound system (VisualSonics Inc., Toronto, Canada) with RMV706 and RMV711 scanheads (see Table 1 for the specifications). The depth of scanning was about $1 \mathrm{~mm}$ which corresponded to 512 signal samples. While the tissue and the probe were fixed in place, we continually acquired 500 ultrasound A-lines (frame rate: $60 \mathrm{fps}$ ) from one spot of the tissue. In other words, we formed a time series of length 500 from each sample of the ultrasound A-line. With each probe type, we acquired two separate lines of RF time series from two different areas of each tissue type.

Table 1. Specifications of the high frequency ultrasound scanheads

\begin{tabular}{c|c|c|c}
\hline Model & Broadband frequency & Center frequency & Axial resolution \\
\hline \hline RMV711 & Up to $82.5 \mathrm{MHz}$ & $55 \mathrm{MHz}$ & $30 \mu$ \\
RMV706 & Up to $60 \mathrm{MHz}$ & $40 \mathrm{MHz}$ & $40 \mu$ \\
\hline
\end{tabular}

\subsection{Feature Extraction}

In this study, tissue types were characterized by the average of FDs computed for all the RF time series in a Region of Interest (ROI). Our high frequency data was acquired in A-mode. Therefore, ROIs were simply segments of RF lines (each signal sample covered a depth of almost 2 microns).

FD of time series originating from natural processes has been extensively studied as a parameter that quantifies nonlinear internal dynamics of complex systems [910]. In such systems, the mechanisms of interaction that give rise to the output time series are not well understood. FD has been shown to have low sensitivity to the noise-induced variations [11. In RF time series analysis, the microstructural information is received along with noise-related variations. Therefore, we chose FD to characterize the RF time series. We used Higuchi's algorithm [12] for computation of the FD of time series which can be summarized as follows: Each sample of the RF data forms a time series $\{X(1), X(2), \ldots, X(N)\}$ 


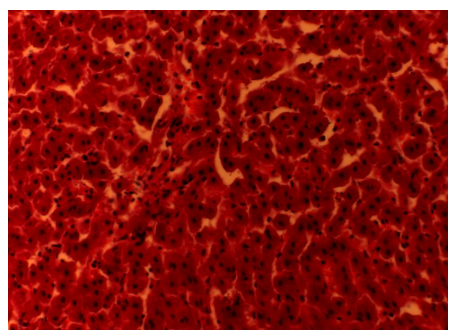

(a) Bovine liver

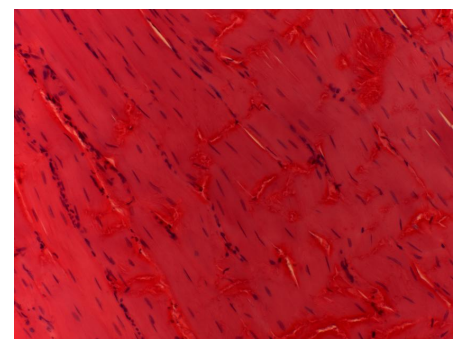

(c) Chicken breast

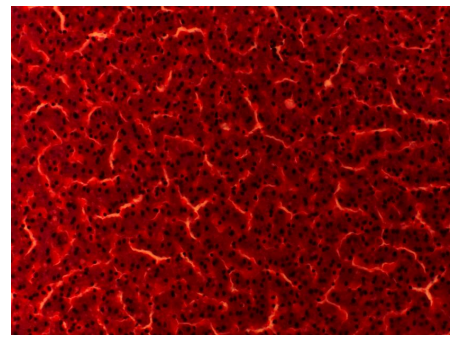

(b) Pig liver

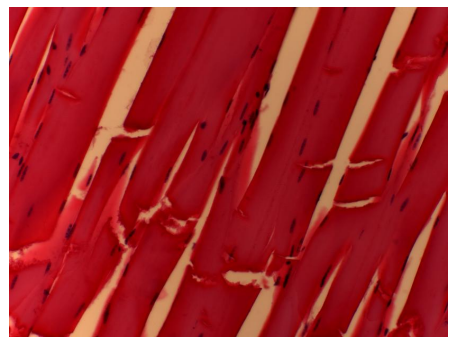

(d) Bovine muscle

Fig. 2. Images of the cellular structure of tissue types used in this study at 200X magnification (acquired from H\&E stained slides with a Zeiss AxioImager M1 microscope)

over sequential ultrasound frames, where $N=500$ for our high frequency RF data. From this time series, we first construct $k$ new time series of form:

$$
X_{k}^{m}:\left\{X(m), X(m+k), X(m+2 k) \ldots, X\left(m+\left[\frac{N-m}{k}\right] . k\right)\right\}
$$

where $k$ is the sampling time interval (which determines the scale, $k<N$ ) and $m=1,2, \ldots, k-1$. Both $m$ and $k$ are integers. The length of each time series, $L_{m}(k)$, is defined as:

$$
L_{m}(k)=1 / k \times\left(\frac{N-1}{\left[\frac{N-m}{k}\right] . k}\right) \times \sum_{i=1}^{\left[\frac{N-m}{k}\right]}|X(m+i k)-X(m+(i-1) \cdot k)|
$$

The average value of $L_{m}(k)$ over $k$ sets, $L(k)$, is the so-called length of the time series at scale $k$. This procedure is repeated for each $k$ ranging from 1 to $k_{\max }$. A line is fitted to values of $\ln (L(k))$ versus $\ln (1 / k)$ and the slope of this line is considered as the FD. The number of the samples, $N$, and the nature of the time series determine the optimal value of the parameter $k_{\max }$. For the current study, the value of $k_{\max }$ was optimized based on the average classification accuracy acquired. We examined $k_{\max }$ values between 4 and 56 . Feature extraction for each A-line involved computation of FD of 512 time series of length 500 . We call the output of this process an FD vector. 


\subsection{Classification}

All classification results reported in the current paper were acquired with a Bayesian approach. If $\omega_{1}$ and $\omega_{2}$ represent ROIs from two categories of tissue involved in one of our classification experiments, and $x$ represents the feature value of a given ROI (which its category is unknown), Bayes' rule states that the classification can be performed based on the following inequalities:

$$
P\left(x \mid \omega_{1}\right) P\left(\omega_{1}\right) \gtrless P\left(x \mid \omega_{2}\right) P\left(\omega_{2}\right)
$$

$P\left(\omega_{1}\right)$ and $P\left(\omega_{2}\right)$ are a priori probabilities (which can be simply calculated as the ratio of the number of ROIs in each category to the total number of ROIs in the two categories). $P\left(x \mid \omega_{1}\right)$ and $P\left(x \mid \omega_{2}\right)$ are the probability density functions (pdf) of feature values in categories 1 and 2 respectively. We fit a Gaussian pdf to the distribution of the feature in each category.

We followed a leave-10\%-out approach to validate the classification procedure. In other words, we randomly partitioned the data in each category to 10 folds. We evaluated the pdfs on $90 \%$ of the data samples, classified the remaining $10 \%$ based on the evaluated pdfs, and repeated the procedure for all 10 portions of the data. We repeated the whole leave-10\%-out process 200 times (each time with a random partitioning of the ROIs to 10 folds). The mean accuracies and standard deviations reported in our results were recorded over these 200 trials.

\section{$3 \quad$ Results and Discussions}

FD vectors from the same tissue types: The first step in our analysis was to perform one-way ANalysis Of VAriance (ANOVA) tests on pairs of FD vectors from the same tissue types. ANOVA is a statistical test in which the null hypothesis is the equality of means in samples from two different populations. As Table 2 illustrates, when two FD vectors from the same tissue type were compared, the p-values in ANOVA tests were relatively large, also the samples from two lines could not be distinguished (classification accuracies close to $50 \%$ ). The ROI size used for classification was 20 microns (10 samples) and $K_{\max }=16$.

Table 2. Comparison of two FD vectors from two RF lines of one tissue type

\begin{tabular}{c|c|c}
\hline Tissue type & $\begin{array}{c}\text { ANOVA p-value } \\
\text { RMV711 }\end{array}$ & $\begin{array}{c}\text { accuracy in separating ROIs from the two lines } \\
\text { results on RMV711 - mean (STD) }\end{array}$ \\
\hline \hline Bovine liver & 0.47 & $52 \%(3.7)$ \\
Pig liver & 0.007 & $47 \%(3.9)$ \\
chicken breast & 0.0001 & $59 \%(3.1)$ \\
Bovine muscle & 0.68 & $53 \%(4.3)$ \\
\hline
\end{tabular}


Table 3. Comparison and classification of data from different tissue types

\begin{tabular}{|c|c|c|c|c|}
\hline Tissue types & $\begin{array}{c}\text { ANOVA } \\
\text { p-value } \\
(\mathrm{RMV} 711) \\
\end{array}$ & $\begin{array}{c}\text { mean (STD) } \\
\text { Res: } 2 \mu \\
(\text { RMV } 711) \\
\end{array}$ & $\begin{array}{c}\text { mean (STD) } \\
\text { Res: } 20 \mu \\
\text { (RMV711) } \\
\end{array}$ & $\begin{array}{c}\text { mean(STD) } \\
\text { Res: } 20 \mu \\
\text { (RMV706) } \\
\end{array}$ \\
\hline Bovine lix & " & $81.1 \%(2.5)$ & $\overline{992.2 \%(5.8)}$ & $96.9 \%(3.5)$ \\
\hline Bor & 0 & $84.1 \%(2.3)$ & $95.5 \%(4.0)$ & $93.7 \%(5.3)$ \\
\hline $\mathrm{C}$ & 0 & $84.6 \%(2.3)$ & $96.0 \%(4.2)$ & $92.3 \%(5.3)$ \\
\hline Pig liver - 1 & 0 & $89.2 \%(2.1)$ & $98.2 \%(3.1)$ & $90.0 \%(6.4)$ \\
\hline Bovine liv & 0 & $73.7 \%(3.0)$ & $83.7 \%(7.1)$ & $65.1 \%(7.9)$ \\
\hline Chicken breast - bovine muscle & $5.7 \times 10^{-13}$ & $64.1 \%(3.1)$ & $72.2 \%(8.9)$ & $63.1 \%(8.1)$ \\
\hline Average & & $79.5 \%$ & $89.6 \%$ & $83.2 \%$ \\
\hline
\end{tabular}

FD vectors from different tissue types $\left(K_{\max }=16\right)$ : We performed the ANOVA tests on FD vectors of different tissue types (Table 3 ). The p-values were all virtually zero and showed that the vectors were statistically different in all six pairs. Two separate FD vectors from each tissue type, computed from the data acquired on RMV711 scanhead, were available. We combined the two vectors of each tissue type to acquire a single vector of length 1000 and used the Bayesian approach described in the previous section to perform pairwise classifications. The results for these classification trials which were in single RF sample resolution, are reported in column 3 of Table 3 . It is interesting to note that even at this resolution, we were successful in classification when the two involved tissue types were from different microstructural categories (rows 1-4); however, when pig liver was compared with bovine liver (row 5) or the two fibrous tissue types were compared (row 6), the classification at this extremely high resolution produced low accuracies.

Furthermore, we examined the performance of our approach at a lower resolution. We averaged 10 samples of each FD vector to acquire vectors of length 50 (100 after combining the two lines from RMV711). Each element of these vectors represented an ROI of size 20 microns. The results of pairwise classification experiments at this level of resolution are presented in column 4, Table 3 . In general, the classification accuracy is significantly higher in this lower resolution (overall accuracy of $89.6 \%$ on RMV711). The mean accuracy for tissues in different microstructural categories was around 95\% (rows 1-4) and for tissues with similar microstructures was around $80 \%$ (rows 5-6).

For validation purposes, the classification process (at 20 micron resolution) was repeated on a similar dataset that was acquired on scanhead RMV706 (which operates at a lower frequency and axial resolution). The results are reported in column 5, Table 3. In general, the overall outcome declined in comparison with RMV711 data (average over all: 83.2\%). However, the same pattern of performance (excellent on different microstructures, moderate on similar microstructures) was observed. The overall decrease in the classification results can be explained by the lower axial resolution of RMV706. 


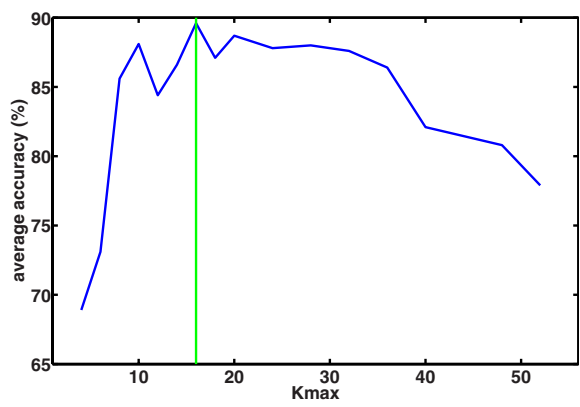

(a)

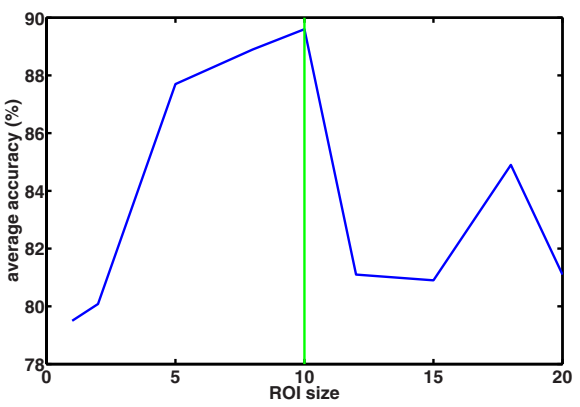

(b)

Fig. 3. (a) The average of classification accuracy over six pairs of tissue for different values of $K_{\max }$ (at resolution of 10 samples). (b) The average of classification accuracy over six pairs of tissue for different number of samples in an ROI $\left(K_{\max }=16\right)$.

Optimal $K_{\max }$ value: We examined different possible values for $K_{\max }$ (or maximum scaling level of the signal) in Higuchi's algorithm. In Figure 3 -a, the average accuracy of tissue classification over six pairs of tissue types is plotted against the values of $K_{\max }$ between 4 and 56 . Values between 10 and 32 resulted in very similar outcomes. The Higuchi algorithm becomes increasingly computationally expensive for large values of $K_{\max }$. We chose $K_{\max }=16$ as a reasonably small number that also resulted in maximum accuracy. This is also in agreement with previous findings about the optimal $K_{\max }$ value on $\mathrm{RF}$ time series acquired from human prostate specimens 7 .

Optimal ROI size: As Figure 3-b illustrates, the classification accuracy increased when ROIs of larger size were used. However, we were limited by the size of the dataset. Increasing the size of the ROI to over 10 samples meant that the Gaussian PDFs were estimated on less than 100 data points and tested on less than 10 points in our leave-10\%-out classification approach. It appears that this low number of samples was not sufficient for training and testing of the classifiers and therefore, we witnessed an unexpected and irregular decrease in the accuracy of classification for ROIs of sizes larger than 10 samples.

Comparison with results at 6.6 MHz: As previously reported, even at frequencies normally utilized on clinical machines $(2-10 \mathrm{MHz})$, the RF time series contain tissue characterizing information [7]6. However, the maximum resolution is much lower. For comparison, we used a Sonix RP (Ultrasonix Inc., Vancouver, Canada) ultrasound machine to collect RF time series at $6.6 \mathrm{MHz}$ from the same specimens that we had scanned at high frequencies. The temporal length of time series (number of frames taken from each cross-section) was 255 and the data was collected with a BPSL9-5/55/10 probe at the rate of 22 frames per second. ROIs of size $8 \times 44 \mathrm{RF}$ samples (equivalent to $0.03 \mathrm{~cm}^{2}$ ) of the tissue were used in classification; 150 ROIs from each tissue type were available. Results reported in Table 4 show an overall accuracy of around $76.5 \%$. 
Table 4. Results of applying the proposed tissue classification approach to the data acquired on a clinical ultrasound machine (probe center frequency: 6.6 MHz)

\begin{tabular}{c|c}
\hline Tissue types & classification accuracy (STD) \\
\hline \hline Bovine liver - chicken breast & $82.9 \%(6.4)$ \\
Bovine liver - bovine muscle & $80.7 \%(6.8)$ \\
Chicken breast - pig liver & $71.4 \%(6.7)$ \\
Pig liver - bovine muscle & $74.8 \%(7.5)$ \\
Bovine liver - pig liver & $69.3 \%(5.3)$ \\
Chicken breast - bovine muscle & $79.6 \%(5.9)$ \\
\hline Average over all six tissue pairs & $\mathbf{7 6 . 5 \%}$ \\
\hline
\end{tabular}

\section{Conclusions}

In this paper we reported the exploitation of high-frequency RF time series for tissue characterization. We used fractal dimension of RF echo time series acquired on ultrasound probes operating at center frequencies of $55 \mathrm{MHz}$ and 40 $\mathrm{MHz}$ to successfully characterize tissue types of different microstructure at the resolution of only 20 microns. The correlation of variations in RF time series with tissue microstructure was evident. FD vectors acquired from different areas of one tissue type were very similar (Bayesian approach resulted in only around $50 \%$ successful separation of ROIs from two areas of one tissue type). For two different tissue types which were from a similar category of microstructure (both mammalian liver or both fibrous muscles), the ROIs could be separated with accuracies around $80 \%$. For tissue types from different microstructural categories, the classification of ROIs based on the Gaussian approach was nearly perfect (up to $98 \%$ accuracy). The same approach was also applied to data acquired on a clinical ultrasound machine and an average accuracy of around $77 \%$ was observed at the resolution of $0.03 \mathrm{~cm}^{2}$.

These findings strongly suggest that the microstructure of the tissue has an effect on the variations of the RF time series. This concept can potentially be used in ultrasound-based detection of pathologic conditions such as cancer.

Acknowledgement. The authors would like to thank Mr. G. Leney from VisualSonics Inc. and Mr. R. Watering for their help in data collection. This work was supported by Natural Sciences and Engineering Research Council of Canada (NSERC), and Institute of Robotics and Intelligent Systems (IRIS).

\section{References}

1. Foster, F.S., Pavlin, C.J., Harasiewicz, K.A., Christopher, D.A., Turnbull, D.H.: Advances in ultrasound biomicroscopy. Ultrasound in Med. \& Biol. 26, 1-27 (2000)

2. Akashi, N., Kushibiki, Dunn, N.C.F.: Acoustic properties of selected bovine tissues in the frequency range 20-200 MHz. J. of Acoust. Soc. Am. 98(6), 3035-3039 (1995)

3. Goss, S.A., Johnston, R.L., Dunn, F.: Compilation of empirical ultrasonic properties of mammalian tissues. II. J. of Acoust. Soc. Am. 68, 93-108 (1980) 
4. Scheipers, U., Ermert, H., Garcia-Schurmann, H.J.S.M., Senge, T., Philippou, S.: Ultrasonic multifeature tissue characterization for prostate diagnosis. Ultrasound Med. Biol. 20(8), 1137-1149 (2003)

5. Lizzi, F.L., Feleppa, E.J., Astor, M., Kalisz, A.: Statistics of ultrasonic spectral parameters for prostate and liver examination. IEEE Transactions on Ultrasonics, Ferroelectrics, and Frequency Control 44(4), 935-942 (1997)

6. Moradi, M., Mousavi, P., Isotalo, P.A., Siemens, D.R., Sauerbrei, E.E., Abolmaesumi, P.: A new approach to analysis of RF ultrasound echo signals for tissue characterization: results of animal studies. In: Proceedings of SPIE conference on Medical Imaging, vol. 6513, pp. 65130P1-65130P10 (2007)

7. Moradi, M., Abolmaesumi, P., Isotalo, P.A., Siemens, D.R., Sauerbrei, E.E., Mousavi, P.: A new feature for detection of prostate cancer based on RF ultrasound echo signals. In: IEEE Ultrasonics Symposium, pp. 2084-2087. IEEE Computer Society Press, Los Alamitos (2006)

8. Thomenius, K.: Evolution of ultrasound beamformers. In: Proc. IEEE Intl Ultras Symp., pp. 1615-1622. IEEE Computer Society Press, Los Alamitos (1996)

9. Accardo, A., Affinito, M., Carrozzi, M., Bouquet, F.: Use of the fractal dimension for the analysis of electroencephalographic time series. Biological Cybernetics 77(5), 339-350 (1997)

10. Henderson, G., Ifeachor, E., Hudson, N., Goh, C., Outram, N., Wimalaratna, Percio, C.D., Vecchio, F.: Development and assessment of methods for detecting dementia using the human electroencephalogram. IEEE Transactions on Biomedical Engineering 53(8), 1557-1668 (2006)

11. Shono, H., Goldberger, C.K.P.A.L., Shono, M., Sugimori, H.: A new method to determine a fractal dimension of non-stationary biological time-serial data. Computers in Biology and Medicine 30(4), 237-245 (2000)

12. Higuchi, T.: Approach to an irregular time series on the basis of the fractal theory. Physica D 31(2), 277-283 (1988) 